\title{
Visual disappearances produced by intensity changes in luminous targets
}

\author{
RICHARD J. ROSE \\ UNIVERSITY OF ILLINOIS
}

Electroluminescent lamps were presented as simple stimuli in para-foveal vision. Both increases and decreases in voltage supplied to the lamps effect perceptual disappearances of the stimuli. Selective fragmentation of multi-unit targets reveals evidence of inhibitory interaction in the visual system.

A suitable stimulus presented in extrafoveal vision under reduced stimulation rapidly loses subjective intensity; with appropriate conditions of voluntary fixation, it will disappear completely. Similar effects occur in the central fovea when the retinal image is effectively stabllized by special techniques. Rapid neutralization of contours and luminous contrast occurs in either case, and the qualitative nature of fadings produced by complex, meaningful targets is very similar. Such evidence suggests that the disappearances produced by these diverse procedures are due to a common underlylng process. This process must involve cell fatigue and the inactivation of neural mechanisms which are responsive to stimulus change (Hebb, 1963; Onley, 1964); stimulation of the retina with patterned lllumination that is truly stationary and of constant intensity produces no evidence of cortical response (Burns, Heron, \& Pritchard, 1962); any moving element in an otherwise stabilized field will remain visible, and a stabllized image which has subjectively disappeared can be reinstated with equal effectiveness by intensity changes in either direction (Iarbus, 1960).

This report describes the perceptual effects of intensity changes in luminous targets presented in parafoveal vision under conditions of reduced stimulation. Results from these studies offer further empirical evidence that the visual system is specialized for transmission of information about stimulus change rather than absolute level of stimulation. Method

Electroluminescent lamps, masked to provide line stimuli of $5^{\prime}$ width and $1^{\circ} 35^{\prime}$ length, were placed in various orientations at $3-8^{\circ}$ eccentricity from a fixation point. Intensity of these stimulus lamps was controlled by a series of voltage regulators, which, in turn, were fed into a constant voltage transformer. Intensity of any stimulus element could be changed according to prearranged schedules by switching that element from one voltage regulator to another. Stimulus intensity was continuously monitored via photocells and an associated oscillograph; subjective disappearances were signalled by a key press and correlated with the photocell record. Lights were mounted on targets affixed to flat black frames and were presented in a darkened room draped to eliminate reflections from other surfaces. In a typical experiment, a dark adapted $\mathrm{S}$ binocularly fixated a spot $6^{\circ}$ below a single line stimulus for a period of 5-30 sec. Initial luminance of the stimulus light was approximately $0.3 \mathrm{ft}-\mathrm{L}$, and the effects of intensity changes, in both directions, of .05 to $.15 \mathrm{ft}-\mathrm{L}$ were tested.

Results

The most striking finding from these experiments is that changes in luminous intensity in either direction produce subjective disappearances. Increments as well as decrements in stimulus intensity effect a sudden and total fading of the stimulus element. Within at least wide limits these effects are not dependent upon the particular orientation, eccentricity, or wave length of the stimulus or the state of dark adaptation of the $S$. And we have been unable to obtain evidence of consistent differences in the nature of visual disappearances whether evoked by increments or decrements in intensity or whether occurring "spontaneously" under voluntary fixation of a constant intensity target.

These phenomena thus appear to be characteristic of extrafoveal perception under reduced stimulation, perhaps due to reciprocal inhibition of antagonistic neurons in the receptive field and the marked specialization of cortical response to spatial or temporal differences in retinal lllumination, rather than to intensity of the illumination itself.

Disappearances are typically of very brief duration, and a faded image can be reinstated by any break in fixation; the act of reporting a disappearance often serves to regenerate the stimulus. In a few Ss disappearances have been prolonged, and for these Ss we have fragmentary evidence that intensity changes in either direction will immediately reinstate the stimulus at normal brightness.

For a given observer the consistency with which intensity changes produce visual disappearances is very high. However, comparative data from different observers reveal large individual differences in the reliability with which disappearances are produced by intensity changes. This is particularly true for increments in intensity. Similar variability is found in the frequency of spontaneous disappearances produced by an unchanging peripheral stimulus. Thus, these differences must reflect subject differences in the ability to maintain voluntary fixation (cf., Clarke, 1957); and subject differences in the readiness to 
Table 1 Percentages of reported disappearances for all elements following intensity changes to entire target

\begin{tabular}{lcccccc}
\hline Target & $\begin{array}{c}\text { Entire } \\
\text { Target }\end{array}$ & Sides & Center & Right & Left & $\begin{array}{r}\text { One Side } \\
\text { \& Center }\end{array}$ \\
\hline A $(|\backslash|)$ & 25.5 & 13.3 & 48.5 & 2.4 & 4.8 & 5.4 \\
B (III) & 70.4 & 4.8 & 4.1 & 4.8 & 8.9 & 6.9 \\
\hline
\end{tabular}

comply and consistently report actual perceptions. But other factors, such as ambient arousal level, may operate as well, and the investigation of these individual differences may prove useful.

Perceptual stability of elements in multi-unit targets reveals evidence of the effects of prior learning, stimulus similarity, and visual field effects (Donderi \& Kane, 1965; McKinney, 1963; Pritchard, Heron, \& Hebb, 1960). The present technique, which studies target stability following intensity changes to any desired combination of target elements, provides a new and powerful approach to further study of such effects. In one preliminary study we have obtained compelling evidence of the reciprocal influence of parallel lines in a three element target. Twelve Ss viewed a target (A) composed of two parallel lines on each side of a center line having a slope of $45^{\circ}$. A second sample of 12 Ss viewed a control target (B) of three parallel lines at the same visual angle. Each $S$ received 18 trials of intensity changes, nine in each direction, according to a standard schedule. Intensity changes were approximately .05, .10, and $.15 \mathrm{ft}-\mathrm{L}$. For both targets the three elements were connected to a single voltage regulator, so that intensity changes occurred simultaneously to the entire target on each trial. Visual disappearances of one or more elements were reported on $76 \%$ of the trials for target $A$ and $67 \%$ of the trials for target B; for both targets disappearances occurred equally often for intensity changes in either direction. Results from this study are summarized in Table 1. A target composed of three parallel lines is organized as a unit. For target B, $70 \%$ of the disappearances evoked by intensity changes are disappearances of the entire target. When fragmentation of this target does occur, any single element or combination of two elements is equally unstable. By contrast, results from joint intensity changes to target $A$ reveal striking evidence of the reciprocal interaction of the two parallel lines; over $60 \%$ of all reported disappearances involve either the center diagonal alone or joint disappearances of the two parallel elements. The center element, when placed in a diagonal orientation, is highly unstable. This is illustrated most clearly when elements of target A are separately controlled; the two parallel lines are run to one set of voltage regulators and the center diagonal element to another. Intensity decreases to the two outside elements reliably leads to perceptual disappearance of the entire target; i.e., the center diagonal consistently fades, although its intensity remains constant. Further, intensity increases to the two parallel lines consistently produce disappearance of the center diagonal. Even sophisticated Ss, advised of the nature of the actual intensity change, report the same phenomenon, that is, sudden disappearance of the center element with no apparent change in intensity level of the two parallel lines.

Such evidence strongly suggests direct inhibitory effects, and further work is now in progress to elucidate these effects.

References

BURNS, B. D., HERON, W., \& PRITCHARD, R. Physiological excitation of visual cortex in cat's unanaesthetized isolated forebrain. $J$. Neurophysiol, 1962, 25, 165-181.

CLARKE, F. J. J. Rapid light adaptation of localised areas of the extrafoveal retina. Opt. Acta, 1957, 4, 69-77.

DONDERI, D. C., \& KANE, E. Perceptual learning produced by common responses to different stimuli. Canad. J. Psychol., 1965, 19, 15-30.

HEBB, D. O. The semiautonomous process: its nature and nurture. Amer. Psychologist, 1963, 18, 16-27.

IARBUS, A. L. Perception of images of variable brightness fixed with respect to the retina of the eye. Biofizika, 1960, 5, 158-161.

McKINNEY, J. P. Disappearance of luminous designs. Science, 1963, $140,403-404$

ONLEY, J. W. Visual sensitivity. Annu, Rev. Psychol, 1964, 15, 29-56.

PRITCHARD, R. M., HERON, W., \& HEBB, D. O. Visual perception approached by the method of stabilized images. Canad. J. Psychol., $1960,14,67-77$.

Note

1. This research was performed during the author's tenure as a National Science Foundation Post Doctoral Fellow at McGill University. I thank D. $\mathrm{O}$. Hebb for his sponsorship and support of this work. 